\title{
ON SPACES OF RIEMANN SURFACES WITH NODES ${ }^{1}$
}

\author{
BY LIPMAN BERS
}

Communicated by Samuel Eilenberg, May 13, 1974

This is a summary of results, to be published in full elsewhere, which strengthen and refine the statements made in a previous announcement [1].

A compact Riemann surface with nodes of (arithmetic) genus $p>1$ is a connected complex space $S$, on which there are $k=k(S) \geqslant 0$ points $P_{1}, \cdots, P_{k}$, called nodes, such that (i) every node $P_{j}$ has a neighborhood isomorphic to the analytic set $\left\{z_{1} z_{2}=0,\left|z_{1}\right|<1,\left|z_{2}\right|<1\right\}$, with $P_{j}$ corresponding to $(0,0)$; (ii) the set $S \backslash\left\{P_{1}, \cdots, P_{k}\right\}$ has $r \geqslant 1$ components $\Sigma_{1}, \cdots, \Sigma_{r}$, called parts of $S$, each $\Sigma_{i}$ is a Riemann surface of some genus $p_{i}$, compact except for $n_{i}$ punctures, with $3 p_{i}-3+n_{i} \geqslant 0$, and $n_{1}+$ $\cdots+n_{r}=2 k$; and (iii) we have

$$
p=\left(p_{1}-1\right)+\cdots+\left(p_{r}-1\right)+k+1
$$

Condition (ii) implies that every part carries a Poincaré metric, and condition (iii) is equivalent to the requirement that the total Poincare area of $S$ be $4 \pi(p-1)$.

From now on $p$ is kept fixed and the letter $S$, with or without subscripts or superscripts, always denotes a surface with properties (i)-(iii). If $k(S)=0, S$ is called nonsingular; if $k(S)=3 p-3, S$ is called terminal.

A continuous surjection $f: S^{\prime} \rightarrow S$ is called a deformation if for every node $P \in S, f^{-1}(P)$ is either a node or a Jordan curve avoiding all nodes and, for every part $\Sigma$ of $S, f^{-1} \mid \Sigma$ is an orientation preserving homeomorphism. Two deformations, $f: S^{\prime} \rightarrow S$ and $g: S^{\prime \prime} \rightarrow S$ are called equivalent if there are homeomorphisms $\varphi: S^{\prime} \rightarrow S^{\prime \prime}$ and $\psi: S \rightarrow S$, homotopic to an isomorphism and to the identity, respectively, such that $g \circ \varphi=\psi \circ f$. The deformation space $D(S)$ consists of all equivalence classes $[f]$ of deformations onto $S$. To every node $P \in S$ belongs a distinguished subset consisting

AMS (MOS) subject classifications (1970). Primary 32G15; Secondary 30A46, $30 \mathrm{~A} 58,14 \mathrm{H} 15$.

${ }^{1}$ Work partially supported by the NSF. 
of all $[f] \in D(S)$ with $f^{-1}(P)$ a node of $f^{-1}(S)$.

We define a Hausdorff topology on $D(S)$ as follows. If $c$ is a closed curve on a part of $S$, denote by $|c|$ the length of the unique geodesic freely homotopic to $S$. Let $C$ be a finite set of closed curves on parts of $S, \epsilon$ a positive number, and $\omega: S^{\prime} \rightarrow S$ a deformation. We say that $\omega$ is $(C, \epsilon)$ small if for every Jordan curve $c^{\prime}$ on a part of $S^{\prime}$ such that $\omega\left(c^{\prime}\right)$ is a node, $\left|c^{\prime}\right|<\epsilon$, and for every $c \in C,|| \omega^{-1}(c)|-| c||<\epsilon$. A set $A \subset D(S)$ is called open if, for every $[f] \in A$, there is a finite set $C$ of closed curves on parts of $f^{-1}(S)$, and a number $\epsilon>0$, such that whenever $\omega: S^{\prime} \rightarrow$ $f^{-1}(S)$ is $(C, \epsilon)$ small, $[f \circ \omega] \in A$.

THEOREM 1. $D(S)$ is a cell. There is an (essentially canonical) homeomorphism of $D(S)$ onto $\mathrm{C}^{3 p-3}$ which takes each distinguished subset onto a coordinate hyperplane.

A deformation $h: S \rightarrow S_{0}$ induces a mapping $h_{*}: D(S) \rightarrow D\left(S_{0}\right)$, called an allowable mapping, which takes each $[f] \in D(S)$ into $[h \circ f]$.

THEOREM 2. Let $S$ and $S_{0}$ have the same genus, and let $k\left(S_{0}\right)=$ $k(S)+l$. If $l=0$, an allowable mapping $D(S) \rightarrow D\left(S_{0}\right)$ is a homeomorphic bijection. If $l>0$, an allowable mapping $D(S) \rightarrow D\left(S_{0}\right)$ is a universal covering of the complement of $l$ distinguished subsets.

The proofs of Theorems 1 and 2 use the so-called Fenchel-Nielsen coordinates (cf. [1, p. 51]). An inequality for Fenchel-Nielsen coordinates stated in [1] as Theorem XV (and previously conjectured by Mumford) implies

THEOREM 3. Let $S_{1}, \cdots, S_{m}$ be all not isomorphic terminal surfaces of genus $p$. There are compact sets $K_{j} \subset D\left(S_{j}\right), j=1, \cdots, m$, such that every $S$ is of the form $S=f^{-1}\left(S_{j}\right),[f] \in K_{j}$, for some $j$.

If $S$ is nonsingular, $D(S)$ can be identified with the Teichmüller space $T_{p}$ of closed Riemann surfaces of genus $p$. For every $S$, each point in $D(S)$, not belonging to a distinguished subset, has a neighborhood which can be naturally identified with a neighborhood in $T_{p}$. Thus an open dense set in $D(S)$ is a complex manifold. It follows that $D(S)$ has the structure of a ringed space.

THEOREM 4. $D(S)$ is a complex manifold which can be realized as a bounded domain in $\mathrm{C}^{3 p-3}$. The distinguished subsets of $D(S)$ are nonsingular analytic hypersurfaces which meet transversally. 
The proof utilizes the Kleinian groups constructed in [1, pp. 46-47] The spaces $X_{\alpha}(S)$ used there are finite ramified coverings of $D(S)$. The following statement is almost obvious.

THEOREM 5. Allowable mappings are holomorphic.

Let $\Gamma(S)$ be the group of allowable self-mappings of $D(S)$ induced by all topological orientation preserving self-mappings of $S$, and let $\Gamma_{0}(S)$ be the subgroup induced by the automorphisms (conformal self-mappings) of $S$. Note that if $\gamma([f])=[g]$ for some $\gamma \in \Gamma(S)$, then $f^{-1}(S)$ is isomorphic to $g^{-1}(S)$. The converse statement is, in general, false.

THEOREM 6. The group $\Gamma(S)$ is discrete, the subgroup $\Gamma_{0}(S)$ is finite and is the stabilizer of [id] $\in D(S)$ in $\Gamma(S)$.

Let $M_{p}$ denote the moduli space (Riemann space) for genus $p$, that is, the set of all isomorphism classes $[S]$ of Riemann surfaces with nodes, of genus $p$. We define a Hausdorff topology in $M_{p}$ by calling a set $B \subset M_{p}$ open if, for every $[S] \in B$, there is a finite set $C$ of closed curves on parts of $S$, and an $\epsilon>0$, such that $\left[S^{\prime}\right] \in B$ whenever there is a $(C, \epsilon)$ small deformation $S^{\prime} \rightarrow S$. The moduli space of nonsingular Riemann surfaces of genus $p$ is known to be a complex space, and is an open dense subset of $M_{p}$. Hence $M_{p}$ has the structure of a ringed space.

There is a canonical mapping $D(S) \rightarrow M_{p}$ which sends $[f] \in D(S)$ into $\left[f^{-1}(S)\right]$.

THEOREM 7. The canonical mapping $D(S) \rightarrow M_{p}$ is holomorphic. Furthermore, [id] $\in D(S)$ has a neighborhood $N$, stable under $\Gamma_{0}(S)$, such that $N / \Gamma_{0}(S)$ is isomorphic to a neighborhood of $[S]$ in $M_{p}$.

Theorems 3 and 7 imply the known (cf. [2])

COROllary (MAYER-MUMFORD). $M_{p}$ is a compact normal complex space (and a V-manifold).

A regular q-differential on $S$ is defined by assigning a holomorphic form $F_{\Sigma}$ of type $(q, 0)$ to each part $\Sigma$ of $S$; the $F_{\Sigma}$ should be either regular at the punctures, or have there poles of order not exceeding $q$, the "residues" at two punctures joined in a node being equal (if $q$ is even) or opposite (if $q$ is odd). The number $\delta(p, q)$ of linearly independent regular $q$-differentials is $p$ if $q=1,(2 q-1)(p-1)$ if $q>1$. If we choose $\delta=$ $\delta(p, q)$ linearly independent $q$-differentials, their "values" at every point of 
$S$, including a node, are the homogeneous coordinates of a point in $\mathbf{P}_{\delta-1}$. In this way one obtains a holomorphic mapping $S \rightarrow \mathbf{P}_{\delta-1}$, the so-called $q$-canonical mapping. This is an embedding for $q>2$ and, in some cases, also for $q=2$ and $q=1$.

THEOREM 8. For every $S$ and every $q \geqslant 1$, there is an analytic hypersurface $\sigma \subset D(S)$, with [id] $\notin \sigma$, and a holomorphic mapping $\Phi$ of $D(S) \backslash \sigma$ into the Chow variety of curves of degree $2 q(p-1)$ in $\mathbf{P}_{\delta(p, q)-1}$ such that, for $[f] \in D(S) \backslash \sigma, \Phi([f])$ is the Chow point of a q-canonical image of $f^{-1}(S)$.

The proof uses the Poincare series described in [1, pp. 48-49]. If $S$ is nonsingular, one knows, from other considerations, that the result is true with $\sigma=\varnothing$. For singular $S$, I could thus far obtain that $\sigma=\varnothing$ only for $q=1$.

\section{REFERENCES}

1. L. Bers, Spaces of degenerating Riemann surfaces, Discontinuous Groups and Riemann Surfaces, Ann. of Math. Studies, no. 79, Princeton Univ. Press, Princeton, N.J., 1974, pp. 43-55.

2. D. Mumford, The structure of the moduli spaces of curves and Abelian varieties, Proc. Internat. Congress Math. (Nice, 1970), Gauthier-Villars, Paris, 1971, pp. 457-465.

DEPARTMENT OF MATHEMATICS, COLUMBIA UNIVERSITY, NEW YORK, NEW YORK 10027 